\title{
The lessons of SARS in Hong Kong
}

\author{
Thomas Sik To Lai and Wai Cho Yu
}

\begin{abstract}
Severe acute respiratory syndrome (SARS) is a novel coronavirus infection which broke out in Hong Kong in March 2003. Princess Margaret Hospital was designated to manage this new, mysterious and serious disease. Healthcare workers had to work under extremely stressful and often risky conditions to care for patients. Despite manpower and equipment reinforcements, staff infection occurred as a result of bodily exhaustion, working in an unfamiliar environment and lapses in infection control. Patients suffered even more, not only due to physical discomfort, but also because of the fear of isolation and death away from family and friends. Health authorities learnt their lessons in the outbreak and formulated emergency plans for future infectious disease epidemics. The healthcare infrastructure has been examined and upgraded with regard to intensive care capacity, infection control measures, professional training, manpower deployment, staff facilities, and stockpiling of drugs and personal protective equipment.
\end{abstract}

KEY WORDS: experience, healthcare workers, outbreak planning, severe acute respiratory syndrome

\section{Introduction}

In January 2003 there were rumours that an outbreak of pneumonic illness was happening in and around Guangzhou, the capital of Guangdong Province, China, and approximately $120 \mathrm{~km}$ northwest of Hong Kong. On 10 February 2003, Guangdong health officials confirmed the outbreak, with a total of 305 cases. Of these, 220 were from Guangzhou and 105 were healthcare workers. There were five deaths. In retrospect it was clear that the situation was understated, but at the time it did serve to alert Hong Kong of a pneumonic illness that was transmissible from human to human, and which can be fatal. ${ }^{1}$ People began stockpiling traditional remedies, notably vinegar, and the Hong Kong Hospital Authority (HA), which manages all public hospitals in Hong Kong, formed a task group to monitor suspicious cases of severe communityacquired pneumonia.

Sporadic cases of atypical pneumonia did begin to appear in Hong Kong. Patients typically present with fever, chills, malaise and a dry cough. Soon the chest radiograph would show reticulonodular infiltrates which often involved the lower lobes initially. Total white cell count was often normal but lymphocyte

Thomas Sik To Lai, consultant physician, Wai Cho Yu, consultant physician

Princess Margaret Hospital, Hong Kong count was typically low. Serum aspartate transaminase level was often mildly elevated. Sputum cultures were negative as were searches for atypical pathogen and respiratory viruses. The disease did not respond to broad-spectrum antibiotics for respiratory pathogens and typically showed progression of lung consolidations. Eventually the majority showed improvement with fever coming down, resolution of lung infiltrates and overall recovery. Others progressed to respiratory failure which required various levels of respiratory support, and some died. ${ }^{2}$ There were small case clusters but clear lines of transmission could not be established. Then on 26 February 2003, a doctor from Guangzhou, who looked after patients with the mysterious pneumonic illness and who have been sick himself, arrived in Hong Kong and checked into a hotel. He transmitted the illness to many people in the hotel and one of those triggered a huge outbreak in a teaching hospital in Hong Kong in early March 2003. Others spread the disease to Singapore, Vietnam, Germany, Canada and elsewhere. The disease became known as severe acute respiratory syndrome (SARS) and in March 2003 the causative agent was discovered to be a novel coronavirus. ${ }^{3}$

\section{Princess Margaret Hospital before the outbreak}

Princess Margaret Hospital (PMH) has a long history of fighting infectious diseases. When it opened in 1975 it was specifically designed to do so, particularly to manage malaria cases that came with the large influx of Vietnamese refugees. By 2003 the refugee problem was long over and PMH had become a major general hospital with 1,300 beds. The infectious disease unit still existed and catered for diseases like varicella, rubella, measles, viral hepatitis, typhoid, malaria, pulmonary tuberculosis, etc. Facilities for airborne isolation were suboptimal. There were no ante rooms as part of the isolation room framework and air changes were similar to general medical wards. Personal protective equipment was also suboptimal, as were infection control practices notably contact precautions. There was no clear-cut preparedness plan for outbreak of infectious disease. Nevertheless, staff members were in general alert for the oncoming pneumonic epidemic. In fact, a patient who had just returned from mainland China and presented to $\mathrm{PMH}$ with severe interstitial pneumonia on 11 February 2003 was immediately transferred to isolation facilities and everyone took extra infection control precautions. He turned out to have been infected by influenza A (H5N1) and for a while led us to believe that the pneumonia epidemic in the mainland was due to the same agent. $^{4}$ 


\section{Impact on $\mathrm{PMH}$, its staff, the patients and their families}

By early March 2003 it became clear that Hong Kong was under the grips of the same pneumonia epidemic that caused so much havoc on the mainland, and the major outbreak around 10 March in a teaching hospital marked the onset of a difficult battle.

The SARS epidemic brought a crisis of exceptional proportion to healthcare workers in Hong Kong who risked their lives caring for SARS patients. The sources of stress came from several dimensions. Firstly, healthcare staff had to prevent getting the illness themselves in order to continue helping others, and personal protective equipment was one of the key issues. N95 masks were reused and labels were attached to identify its owner. A while later this was recognised to be a dangerous practice, hence much larger quantities of masks were called for and there were times when supply was rather tight. Still a little later it was noted that for high-risk procedures, for example endotracheal intubation, eye shields and preferably face shields must be used and again there was a supply problem.

Secondly, SARS is a severe illness with a high proportion of patients going into respiratory failure. They also tended to worsen quickly and such events often happened after a period of clinical improvement. Furthermore, there was no known specific treatment for SARS and the healthcare team facing a worsening patient only had supportive treatment and some unproven treatment (like ribavirin and corticosteroids) in their armamentarium. These were coupled with the large number of SARS patients being cared for at the same time at the height of the epidemic in early April 2003 and it can be imagined that there was tremendous stress to the healthcare team.

The physical and intellectual demands on healthcare workers were further aggravated by a third factor: psychological stresses not only from the fear of vulnerability, because of the increasing number of healthcare workers infected, but also from the emotional torment of seeing the anguish of sick colleagues. Additionally, staff were worried that if they get the illness they might transmit it to their family members. This was despite early observation that there was lack of onward transmission if subjects were isolated once they developed symptoms (and later confirmed by viral shedding studies ${ }^{5}$ ). Many chose to stay away from home and shun social gatherings and this compromised the much needed family and social support during these hard times.

SARS patients fared worse. They were sick, weak and breathless, and there was always fear of the illness worsening. There were also problems with treatment side effects. One patient was suspected to suffer from manic depressive psychosis, when in fact he was manic after receiving steroid injections and depressive after receiving ribavirin. Symptoms promptly disappeared when these drugs were stopped. All these torments have to be endured in isolation because patients were denied the comfort of having visits by family members and friends, who were enormously concerned but could do very little.

Non-SARS patients also suffered. Hospital beds and other healthcare resources were diverted to treat the epidemic. Elective procedures, including surgical operations, were put on hold. Additionally this group of patients were afraid that they would get SARS if they stayed in hospital or paid clinic visits. The levels of medical care they received were therefore compromised.

\section{Coping with a high proportion of staff who were ill}

Facing the high concentration of SARS patients and workload pressure, a significant number of staff contracted SARS. When the epidemic ended in early June 2003, there were 1,755 confirmed cases of SARS in Hong Kong, with 384 healthcare workers among them. Eight healthcare workers succumbed to the disease, including four doctors, one nurse, two healthcare assistants and one ward attendant. The healthcare workers had deservedly gained the community's highest salute for their sacrifice, dedication, courage and professionalism.

In the war against SARS, all HA staff members were expected to, for ethical and altruistic reasons, prepare for deployment outside their existing areas where necessary to cope with the work demand and relieve sick colleagues. Take PMH, the territory's designated SARS hospital, as an example: a total of 76 medical and nursing staff had been deployed to PMH from other hospitals through central coordination by the HA. Some of them joined the rescue on a voluntary basis.

When PMH was designated as the SARS hospital, there was a sudden influx of patients to the intensive care unit (ICU), which became the worst hit area. To deal with this heavy workload, staff from the operating theatre, coronary care unit, high dependency unit and respiratory ward was deployed to the ICU. Significant help was also offered by ICUs of other hospitals. A total of 20 doctors and 42 nurses from all sources were deployed to work in PMH ICU. Despite the additional manpower, staff working in an unfamiliar environment was inefficient and ineffective. There were concerns about efficacy of infection control measure in the face of high patient concentration. Many healthcare workers acquired the infection through high-risk procedures such as intubation, resuscitation and waste handling, while for others the route of infection was less obvious and presumably occurred through contact with fomites. Intensive training sessions in infection control were held for newcomers to better equip them to protect themselves. With the introduction of effective infection control measures as well as administrative arrangements to decrease the number of ICU patients, staff infection rates started to drop. Asymptomatic infection of hospital-based healthcare workers was distinctly rare. ${ }^{6}$

A dedicated team was formed to work in SARS wards of $\mathrm{PMH}$ in order to avoid exposure of all staff to SARS and minimise the number infected. Temporary accommodations from within PMH and external sources were offered to all staff, especially those of the SARS team, so that they can have the choice to stay away from home after duty. A special clinic was established for staff and their relatives. All staff with fever would be isolated and followed-up daily. Single rooms in a sick bay for staff requiring temporary isolation and recovery quarters for staff discharged from SARS wards were arranged. Wards with 
private room facilities were reserved for staff suspected or confirmed to be suffering from SARS.

Despite the many measures to allay anxiety and boost morale the stress proved too much for some staff. The HA very thoughtfully devised psychological support programmes for staff during the SARS crisis.

\section{How our experience has helped in planning for future outbreaks}

Had the sick and febrile doctor from the mainland been identified at the border and sent to a hospital immediately, Hong Kong and some other parts of the world might have been spared serious outbreaks. SARS has underlined the need for better monitoring of the situation and early identification and isolation of potentially infectious subjects to contain future outbreaks of new respiratory infectious diseases.

At the peak of SARS with a rapidly evolving scenario, Hong Kong's health authorities had no well-defined response plan and the situation had been likened to building a boat and sailing it in uncharted waters at the same time. The onslaught of SARS was a tragic lesson that mandated the health administration to formulate emergency preparedness plans for the unprecedented and unexpected events of infectious disease epidemics, which could lead to grave and far-reaching consequences. The surge capacity of the healthcare infrastructure was closely examined, especially with regard to intensive care capacity; infection control measures, professional training, manpower deployment, staff facilities, and stockpiling of drugs and personal protective equipment. The end product, which is the crisis management plan, calls for timely information sharing, building up of communication capabilities and creation of more up-to-standard isolation rooms. It also emphasises that the contingency response should be centrally coordinated with clear lines of authority, command and control. Through their concerted efforts against SARS, Hong Kong's healthcare workers have developed a sense of teamwork, communication and trust which has never been stronger.

There have been concerns on the rather haphazard use of unproven treatments of doubtful clinical efficacy and with potential short- and long-term side effects. ${ }^{7,8}$ Task groups were formed after SARS to discuss procedures for trial use and subsequent development of specific treatments with potential clinical benefits.

Despite all the technical advances in recent years, we have now gone back to basics in infection control routines and emphasise commonsense behaviour, like frequent hand washing and avoidance of touching the face, etc. In preparation for the war against possible emerging epidemics, we have invested heavily on infectious disease and infection control education, training, practice and research and development during peace time. As infectious diseases know no geographical boundaries, their effective control requires a strong global collaborative partnership involving the Ministries of Health of different countries, the World Health Organization and other international bodies.
The discovery that the SARS coronavirus originated from bats should serve to remind us that the ever increasing human population is dangerously encroaching on the natural environment. ${ }^{9}$ Add this to the lifestyle of consuming game animals (such as masked palm civets) and living close to domestic livestock (such as poultry) and novel zoonotic infections would be invited to our doorsteps.

\section{Conclusion and looking ahead}

There are at least six lasting lessons that can be learned from SARS:

- Close collaboration between medicine and science can solve the mystery of an unknown but threatening infectious agent.

- The response and resilience of different parts of the healthcare system have been tested, making the authorities better prepared for the anticipated and perhaps much more serious next pandemic.

- We are affected by the situations, ranging from living conditions, daily life practices and healthcare standards, in other countries, especially the less developed ones.

- SARS has shown the healthcare administrators the importance of timely institution of safety procedures to protect the frontline staff.

- Even in dire circumstances, principles on use of novel treatments must be adhered to.

- In contrast to the usual societal value of financial benefit and everyone-for-themselves, healthcare workers have shown amazing heroism and dedication in caring for patients with SARS at dire personal risks and have thus upheld the ethical principle of the profession.

Will SARS come back? The balance of opinions among experts is that SARS may return as sporadic cases, but it is improbable for it to reappear in such an epidemic scale. The recent H1N1 pandemic, although its case-fatality rate is not as high as SARS, should be taken very seriously. Newly learned lessons will be put to good use and the young preparedness plans will have the chance to prove their worth.

\section{References}

1 Zhong NS, Zheng BJ, Li YM et al. Epidemiology and cause of SARS in Guangdong, Peoples' Republic of China, in February 2003. Lancet 2003;362:1353-8.

2 Leung CW, Lai TST. Pediatric and adult SARS. In: Chin R (ed), Emergency management of infectious diseases. New York: Cambridge University Press, 2008.

3 Peiris JSM, Lai ST, Poon LLM et al. Coronavirus as a possible cause of severe acute respiratory syndrome. Lancet 2003;361:1319-25.

4 Peiris JS, Yu WC, Leung CW et al. Re-emergence of fatal human influenza: a subtype H5N1 disease. Lancet 2004;363:617-9.

5 Cheng PK, Wong DA, Tong LK et al. Viral shedding patterns of coronavirus in patients with probable severe acute respiratory syndrome. Lancet 2004;363:1699-700.

6 Lai TST, Ng TK, Seto WH et al. Low prevalence of subclinical severe acute respiratory syndrome-associated coronavirus infection among 
hospital healthcare workers in Hong Kong. Scand J Infect Dis

2005;37:500-3.

7 Yu WC, Hui DSC, Chan-Yeung M. Antivirals and corticosteroids in the treatment of severe acute respiratory syndrome (SARS). Thorax 2004;59:643-5.

8 Lai ST. Treatment of severe acute respiratory syndrome. Eur J Clin Microbiol Infect Dis 2005;24:583-9.

9 Li W, Shi Z, Yu M et al. Bats are natural reservoirs of SARS-like coronaviruses. Science 2005;310:676-9.

Address for correspondence: Dr T Sik To Lai, Department of Medicine and Geriatrics, Princess Margaret Hospital, 2-10 Princess Margaret Hospital Road, Lai Chi Kok, Kowloon, Hong Kong. Email: laist@ha.org.hk 